\title{
A Machine Vision System for Inspecting Bearings
}

\author{
Jože Derganc, Franjo Pernuš \\ University of Ljubljana \\ Faculty of Electrical Engineering \\ Tržaška cesta 25, 1000 Ljubljana, Slovenia \\ E-mail: joze.derganc@fe.uni-lj.si,feri@fe.uni-lj.si
}

\begin{abstract}
In this paper we describe a machine vision system for inspecting bearings, which are an important part of electro-mechanical $\mathrm{kWh}$ meters. The system consists of a personal computer with a frame grabber, a black and white progressive scan CCD camera, and a mechanical device with a stepper motor controlled by a special controller connected to the RS232 port of the personal computer. The quality of a bearing depends on the eccentricity of the needle and the length of the needle that extends out of the cylinder. These two parameters are robustly and accurately defined by the Hough transform and the regression methods. The presented results show that the proposed machine vision system allows accurate, reproducible, and robust $100 \%$ inspection of bearings of electro-mechanical kWh meters and as such it may be a valuable tool for ensuring highend-product quality.
\end{abstract}

\section{Introduction}

The driving force in today's manufacturing environment are: quality improvement, cost reduction increased volume, and shorter cycle times for manufacturing [1]. Improved quality is the key factor in satisfying the demands on modern manufacturing. The quality of many raw materials, parts, and products can be measured through electrical or mechanical means and through visual inspection. Inspection by eye is costly, subjective, qualitative, inaccurate, eye-straining, and time-consuming. Manufacturers therefore look for fast, accurate, reliable, and consistent automated visual inspection of their products to reduce manual involvement in the application of pass/fail criteria.

During the last two decades, machine vision systems, which perform automated visual inspection, have been applied slowly but surely to a variety of manufacturing challenges, all with the goal of improving quality and productivity in the manufacturing process $[1,2,3,4,5]$. Machine vision unifies illumination, imaging, image processing and analysis, and material handling technologies to provide non-contact location, characterization, and manipulation of stationary or moving objects. It uses features such as distance, size, shape, colour, etc. to discriminate between good and defected objects. Machine vision systems operate in constrained and controlled environments in which there is control over illumination, image contrast and resolution, position and orientation of objects, or knowledge about the objects to be inspected. This facts can be exploited by feature detection algorithms which lead to fast, precise and reproducible inspection. Usually high speed, high quality and high resolution applications require innovative, customised solutions, outside the scope of standard "of the shelf" systems. In this paper we describe such a machine vision system for inspecting bearings which are an important part of electromechanical kWh meters.

\section{Problem description}

The upper bearing, to which the main shaft with the aluminium plate, which rotates under the electromagnetic effects produced by the current and voltage, is attached is a vital part of an electro-mechanical kWh meter (Figure $1)$.

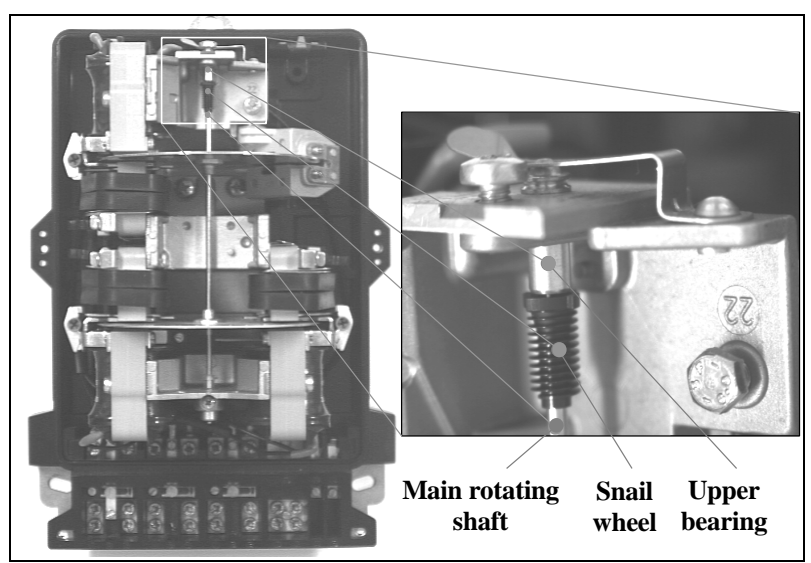

Figure 1: Position of the upper bearing in an electromechanical kWh meter

The upper bearing is a hollow cylinder with a needle in the center that is fixed to the bottom (Figure 2). The quality of the upper bearing depends on:

a) the eccentricity of the needle,

b) the length of the needle that extends out of the cylinder. 
The eccentricity, which may be visualised by rotating the bearing (Figure 3), is defined as the distance between lines ne and ref, the lines of symmetry of the needle and a cylinder, respectively (Figure 4). The points PQRS, $\mathrm{PS} \| \mathrm{QR}, \mathrm{P}$ and $\mathrm{Q}$ on line $n e$ and points $\mathrm{R}$ and $\mathrm{S}$ on line ref, form a trapezoid, whose median has been choosen to define the distance between the lines. The median thus represents the eccentricity $e$ in a certain position of the bearing. The eccentricity $E$ is the largest median $e$ of a number of trapeziods obtained during rotation. The height of any trapezoid represents the length $d$ of the needle that extends out of cylinder. The eccentricity $E$ of a good bearing must be less than $0.1 \mathrm{~mm}$, while the length $d$ of the needle that must be $1.2 \mathrm{~mm} \pm 0.1 \mathrm{~mm}$.

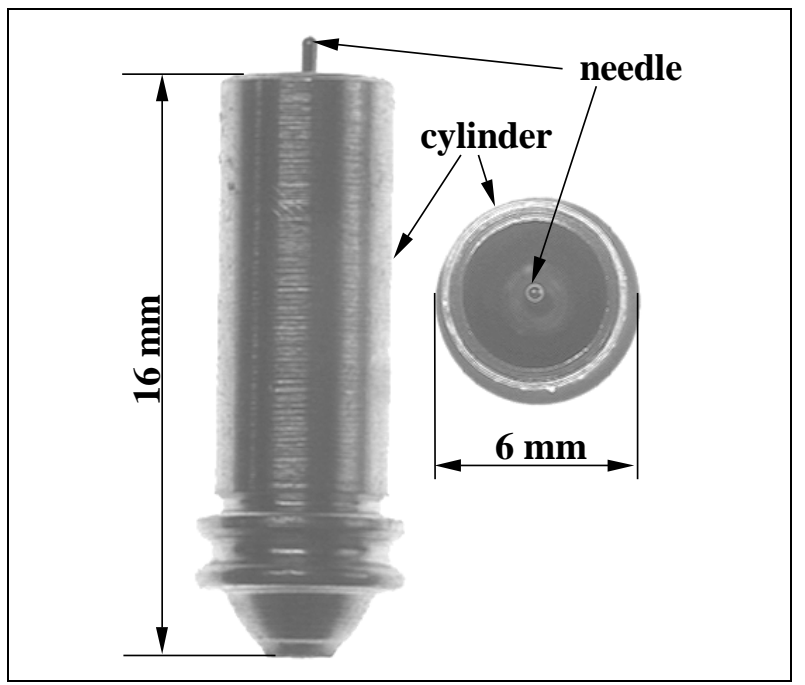

Figure 2: Upper bearing; side view (left), view from above (right)

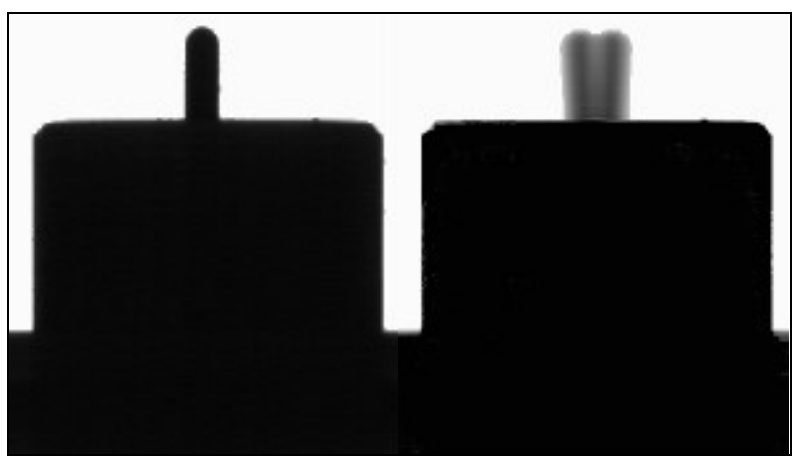

Figure 3: A straight (left) and an eccentric bearing (right) during rotation

The edges of a bearing are all straight lines, except at the top of the needle where edge points form a semiellipse. The parameters of the straight lines and the semiellipse, which are used to find the points PQRS of a trapezoid and thus the eccentricity and the length of a needle have to be obtained by fast and robust algorithms, which at the same time must give accurate results. Dust and other small particles on the bearing should not affect the measurements.

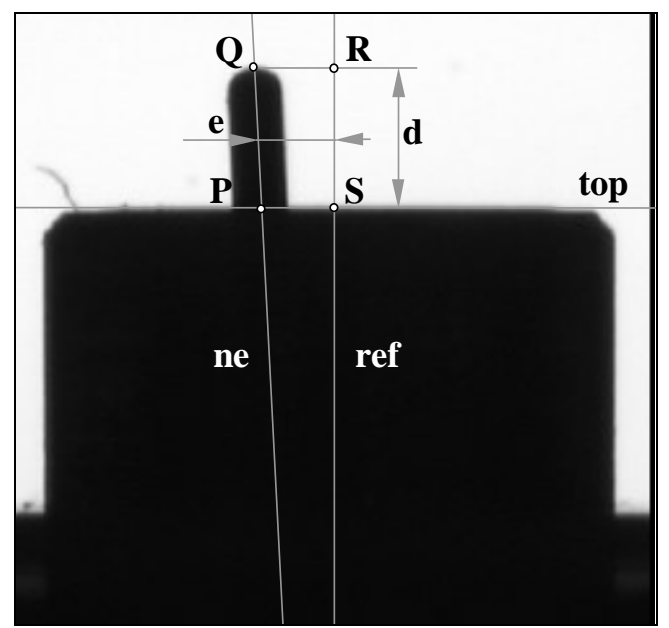

Figure 4: Eccentricity $e$ of a bearing and the length $d$ of the needle extending out of the cylinder

\section{The machine vision system}

The machine vision system for measuring the eccentricity and the length of the needle extending out of the cylinder consists of a personal computer (PC), a black and white progressive scan CCD camera, illumination, and a mechanical device with a stepper motor controlled by a special controller connected to the RS232 port of the PC (Figure 5). The system allows accurate non-contact measurements while the bearing is rotated.

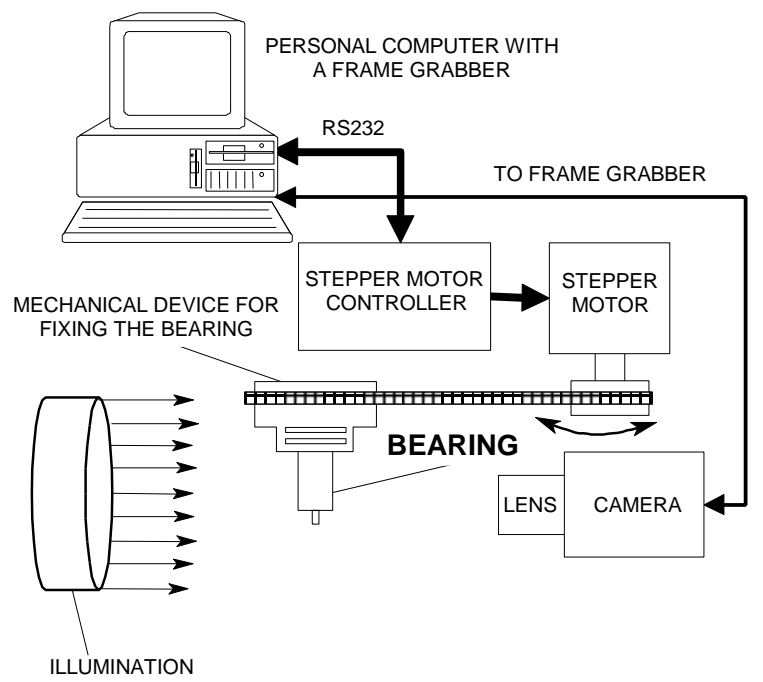

Figure 5: Outline of the machine vision system for inspecting bearings

\section{Methods for visual inspection}

Figure 6 shows the flow chart of the bearing inspection procedure. After a bearing is set in its position in front of a camera, it is rotated and during rotation for 360 degrees $N$ images are taken, each image showing 
the bearing rotated for $k * 360 / N, k=0,1,2, \ldots, N-1$, degrees. In each image the differential edge operator is used to extract those image points which have a high likelihood of being on or near the straight lines or the semiellipse. The Hough transform (HT), which is inherently robust against noise, is then used to determine the parameters of straight lines. Because the HT demands considerable computation and one of the major constraints on the inspection is lack of time, accuracy is first sacrified to speed. The parameters of the straight lines are only approximatively defined. The accuracy is then improved by yet another step of processing. A line fitting procedure is added to refine the output of the HT procedure. In the following subsection a more detailed description of the algorithm is given.

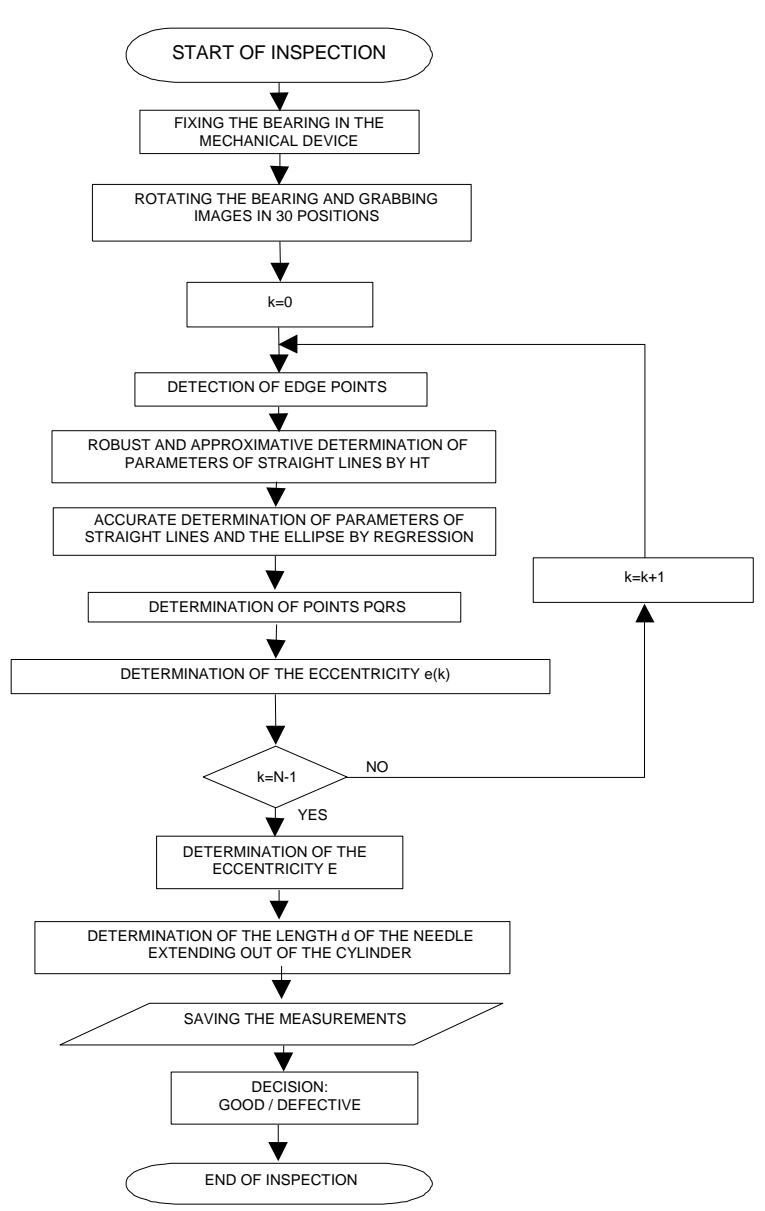

Figure 6: Flow chart of bearing inspection

\subsection{Determination of parameters}

Because the system operates in a constrained and controlled environment, intensity profiles obtained along 7 predetermined lines (Figure 7) can be used to determine 6 regions of interest (ROI) (Figure 8). In ROIs A-E the edges of a bearing are straight lines while in region $F$ the edge forms a semiellipse. To proceed with line detection, we first apply the Sobel operator [6] to derive the edge magnitudes $G\left(x_{i}, y_{i}\right)$ at each pixel $\left(x_{i}, y_{i}\right)$ :

$G\left(x_{i}, y_{i}\right)=\sqrt{g_{x}^{2}\left(x_{i}, y_{i}\right)+g_{y}^{2}\left(x_{i}, y_{i}\right)}$

where $g_{x}$ and $g_{y}$ represent the partial gradients in the $x$ and $y$ direction, respectively. Second, in each ROI only the pixels $\left(x_{i}, y_{i}\right)$ for which:

$G\left(x_{i}, y_{i}\right)>G_{\max } / 2$

where $G_{\max }$ is the highest gradient magnitude in a ROI, are kept. Next, the Hough transform and regression methods are used to determine the edges to sub-pixel precision.

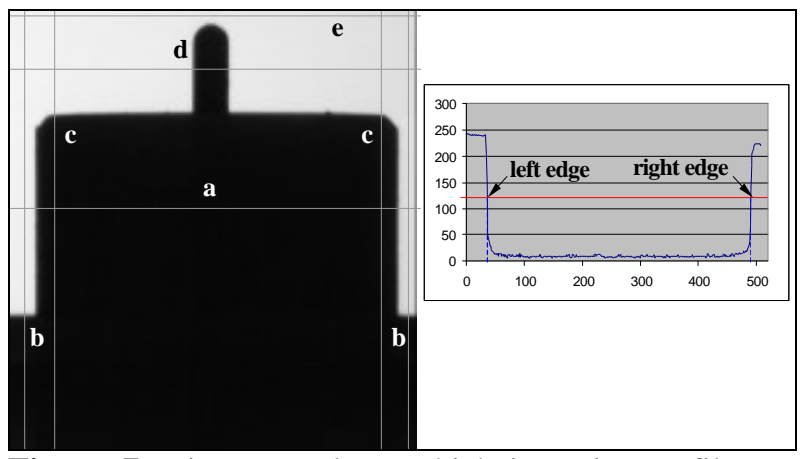

Figure 7: Lines a-e along which intensity profiles are determined. The intensity profile along line $\mathrm{a}$ is given on the right side

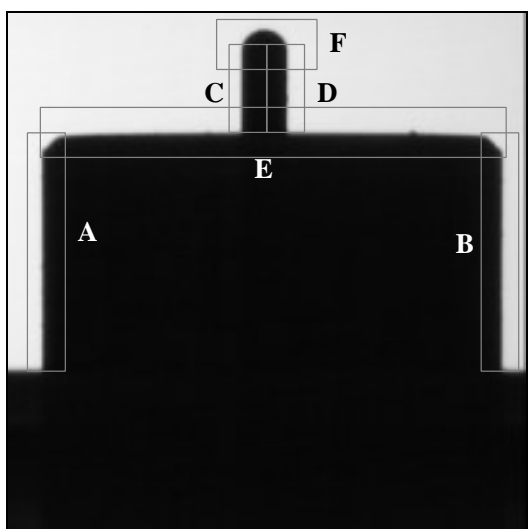

Figure 8: Regions of interest (A-E) in which we search for straight lines and region $F$ in which a semiellipse is searched for

Each straight line in ROIs A-D may be parametrically described as:

$x=\tilde{m} y+\tilde{c}$

while the horizontal straight line in ROI E is defined as:

$y=m x+c$ 
In each ROI the approximative values of the parameters $m$ and $c$ and $\tilde{m}$ and $\tilde{c}$ are obtained by the Hough transform [6]. In a ROI the edge points $\left(x_{i}, y_{i}\right)$ for which:

$$
\frac{\left|x_{i}-\tilde{m} y_{i}-\tilde{c}\right|}{\sqrt{1+\tilde{m}}}<d_{\max }
$$

or

$$
\frac{\left|y_{i}-m x_{i}-c\right|}{\sqrt{1+m}}<d_{\max }
$$

where $d_{\max }$ is a predefined threshold ( $d_{\max }=3$ pixels in our implementation), are further used to precisely define the parameters $\tilde{m}$ and $\tilde{c}$ by linear regression $[7,8]$ :

$$
\begin{gathered}
\tilde{m}=\frac{\sum_{i=1}^{M} y_{i}^{2} \sum_{i=1}^{M} x_{i}-\sum_{i=1}^{M} y_{i} \sum_{i=1}^{M} x_{i} y_{i}}{M \sum_{i=1}^{M} y_{i}^{2}-\sum_{i=1}^{M} y_{i} \sum_{i=1}^{M} y_{i}} \\
\tilde{c}=\frac{M \sum_{i=1}^{M} x_{i} y_{i}-\sum_{i=1}^{M} x_{i} \sum_{i=1}^{M} y_{i}}{M \sum_{i=1}^{M} y_{i}^{2}-\sum_{i=1}^{M} y_{i} \sum_{i=1}^{M} y_{i}}
\end{gathered}
$$

where $M$ is the number of edge points which satisfy Eq. 5 or 6 . Similar equations are used to accurately define the parameters $m$ and $c$.

The parameters $\left(\tilde{m}_{A}, \tilde{c}_{A}\right)$ and $\left(\tilde{m}_{B}, \tilde{c}_{B}\right)$ of the straight lines in ROIs $A$ and $B$, respectively, representing the left and the right border of the cylinder are used to determine the line ref:

$$
x_{r e f}=\frac{\tilde{m}_{A}+\tilde{m}_{B}}{2} y_{r e f}+\frac{\tilde{c}_{A}+\tilde{c}_{B}}{2}
$$

The line $n e$ is defined as:

$$
x_{n e}=\frac{\tilde{m}_{C}+\tilde{m}_{D}}{2} y_{n e}+\frac{\tilde{c}_{C}+\tilde{c}_{D}}{2}
$$

where $\left(\tilde{m}_{C}, \tilde{c}_{C}\right)$ and $\left(\tilde{m}_{D}, \tilde{c}_{D}\right)$ are the parameters of the straight lines in ROIs C and D, respectively, representing the left and the right border of the needle. The line top representing the upper border of the cylinder is defined as:

$$
y_{\text {top }}=m_{E} x_{\text {top }}+c_{E}
$$

The parameters of the semielliptic tip of a needle, lying in region $\mathrm{F}$, are obtained by the regression method
[8,9] using $R$ points $\left(x_{i}, y_{i}\right)$ which satisfy Eq. 2. The equation of an ellipse $Q(x, y)$ is:

$Q(x, y)=a_{1} x^{2}+a_{2} x y+a_{3} y^{2}+a_{4} x+a_{5} y+1=0$

and

$Q\left(x_{i}, y_{i}\right)=a_{1} x_{i}^{2}+a_{2} x_{i} y_{i}+a_{3} y_{i}^{2}+a_{4} x_{i}+a_{5} y_{i}+1$

is the algebraic distance of point $\left(x_{i}, y_{i}\right)$ to the ellipse. The parameters of the ellipse best fitting the $R$ edge points are obtained by minimising:

$$
S E=\sum_{i=1}^{R} Q\left(x_{i}, y_{i}\right)^{2}
$$

Obtaining 5 partial derivatives of $S E$ with respect to $a_{l}$, $a_{2}, a_{3}, a_{4}$ and $a_{5}$, and setting these partial derivatives to zero provides five simultaneous equations. For $a_{l}$ the equation is:

$$
\begin{aligned}
& \frac{\partial S E}{\partial a_{1}}=\sum_{i=1}^{R} 2 x_{i}^{2}\left(a_{1} x_{i}^{2}+a_{2} x_{i} y_{i}+a_{3} y_{i}^{2}+a_{4} x_{i}+a_{5} y_{i}+1\right)= \\
& =2\left(a_{1} \sum_{i=1}^{R} x_{i}^{4}+a_{2} \sum_{i=1}^{R} x_{i}^{3} y_{i}+a_{3} \sum_{i=1}^{R} x_{i}^{2} y_{i}^{2}+\right. \\
& \left.\quad+a_{4} \sum_{i=1}^{R} x_{i}^{3}+a_{5} \sum_{i=1}^{R} x_{i}^{2} y_{i}+\sum_{i=1}^{R} x_{i}^{2}\right)=0
\end{aligned}
$$

The solution to the 5 equations gives the parameters of the ellipse $Q(x, y)$ which minimises the error function SE.

\subsection{Eccentricity and length of the needle}

The equations of the straight lines ne, ref and top and the ellipse are used to define the points $\mathrm{P}, \mathrm{Q}, \mathrm{R}$, and $\mathrm{S}$. The eccentricity $e(k)$, in position $k, k=0,1, . . N-1$ is determined as the median of the trapezoid formed by these points:

$$
e(k)=K_{C} \frac{\overline{P S}+\overline{Q R}}{2}
$$

where $K_{C}$ is the calibration coefficient obtained during calibration of the system with a bearing of known dimensions.

Figures 9 a-c show how the eccentricities $e(k)$, $k=0,1,2, \ldots, 29$, change during rotation of four bearings with the highest eccentricity $0.13,0.05$ and $0.03 \mathrm{~mm}$, respectively. One can observe that the eccentricity values form a discrete sine function.

Its complex discrete spectrum $E^{d}(m)$ (Figure 10) may be obtained by the Discrete Fourier transform (DFT) [8]:

$$
E^{d}(m)=\sum_{k=0}^{N-1} e(k) e^{-j \frac{2 \pi}{N} m k} \quad m=0 . . N-1 \quad k=0 . . N-1
$$




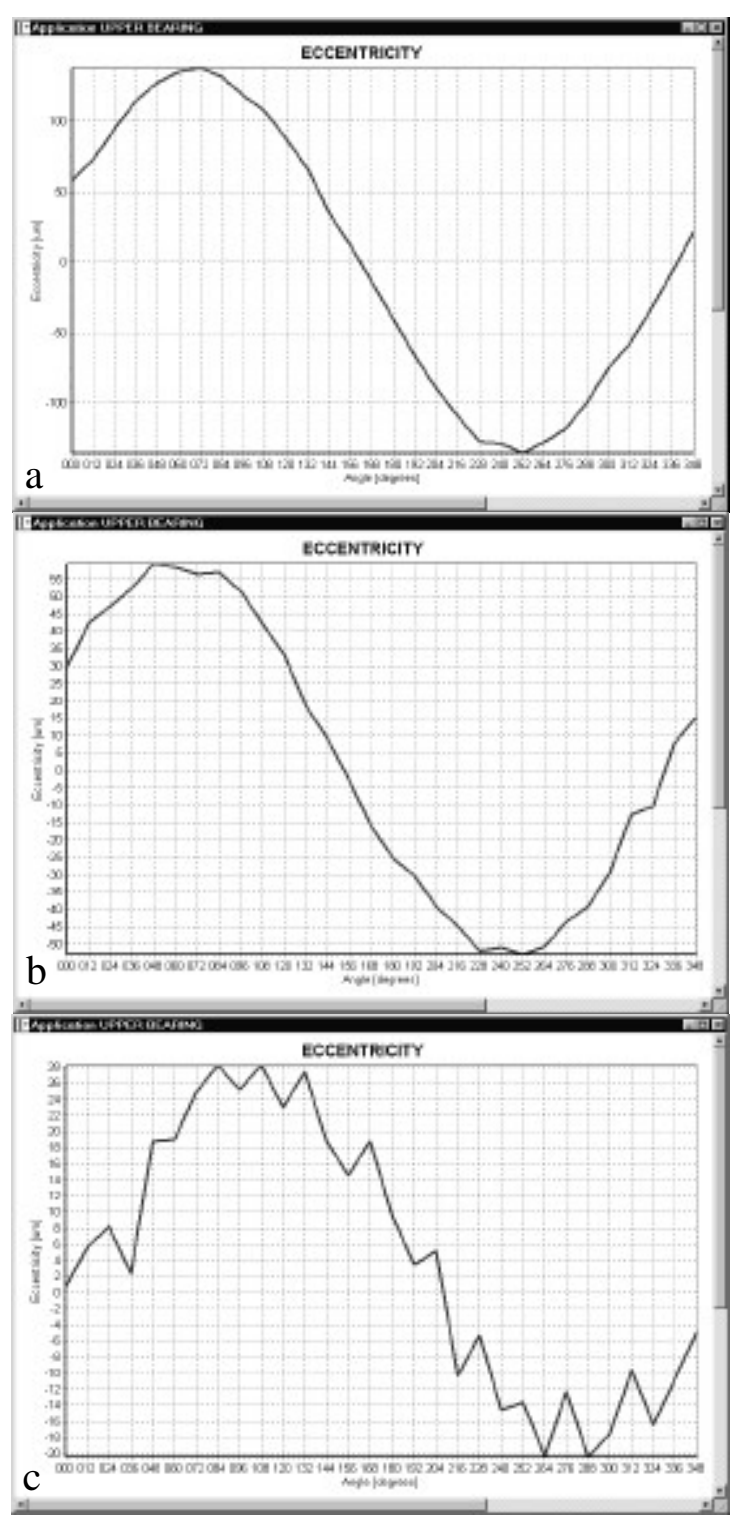

Figure 9: Eccentricities $e(k), k=0,1,2, \ldots, 29$, of four bearings

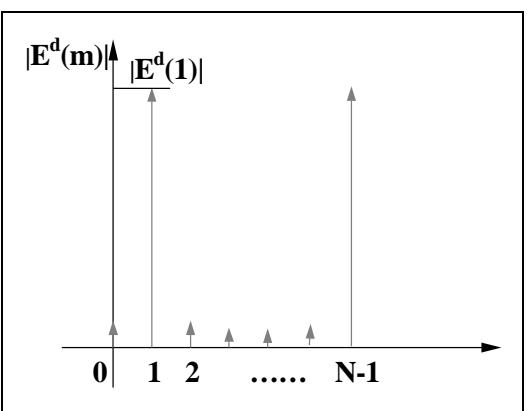

Figure 10: Absolute values of complex discrete spectrum $\left|E^{d}(m)\right|$

The largest eccentricity $\mathrm{E}$ of a bearing is obtained by $E^{d}(1)$ :

$E=\frac{2}{N}\left|E^{d}(1)\right|$
The length $d$ of the needle extending out of the cylinder is the distance between points $\mathrm{R}$ and $\mathrm{S}$ :

$$
d=K_{C} \overline{R S}
$$

\section{Results}

To test the influence of the number of images taken per rotation $(N)$ on the value of the eccentricity $E$, this was determined from $N=3,5,10,15,20,25$ and 30 views. At the same time the reproducibility of the measurements for each $N$ was tested by starting the rotation of a bearing from 50 randomly selected positions. For each $N$ the standard deviation of 50 measurements was defined. The results presented in Figure 11 indicate that the measurements are highly reproducible if the eccentricity is defined from more than $10(N>10)$ positions of the bearing. Because speed is a crucial factor, the machine vision system uses only 15 images to determine the eccentricity of a bearing.



Figure 11: Reproducibility of the measured eccentricity

To justify the sequential use of the Hough transform followed by linear regression we show the results obtained by the proposed method and by hard redescenders M-estimators method [10] on a bearing with dust particles on it (Figure 12).

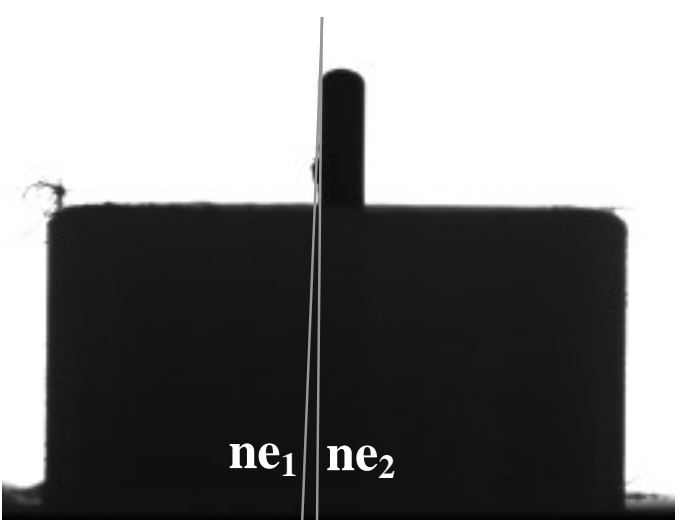

Figure 12: Two lines, $\mathrm{ne}_{2}$ detected by the Hough transform followed by linear regression and $\mathrm{ne}_{1}$ by $\mathrm{M}$ estimators 
The line $\mathrm{ne}_{2}$ is obtained by the combination of the Hough transform and linear regression, while the line ne ${ }_{1}$ is obtained with the M-estimators. It is evident that dust on the needle's left edge hampers the correct detection of the line by M-estimators, because the number of outliers in data exceeds the breakdown point of $33 \%$ for linear regression [11].

\section{Conclusions}

In this paper a machine vision system for inspecting bearings of electro-mechanical $\mathrm{kWh}$ meters has been described. The obtained results show that the methods used to define the eccentricity of a bearing are fast and robust and that they give reproducible results. Such a machine vision system will be incorporated in the electro-mechanical $\mathrm{kWh}$ meters production line. It is expected that it will be a valuable tool for ensuring highend-product quality. In the future we will not only inspect the bearings, but will try also to strengthen the needles of eccentric bearings, by a straightening shaft and a stepper motor (Figure 13).



Figure 13: Possible straightening of eccentric bearing

For this purpose the bearing has to be rotated for $\varphi_{R}$ from the current position $\varphi_{\mathrm{o}}$ to the position $\varphi_{S}$, the position of the maximal decline of the needle to the left (Figure 14):

$\varphi_{R}=\varphi_{S}-\varphi_{o} \quad \varphi_{S}=270^{\circ}$

where $\varphi_{0}$ is the phase angle of the discrete sine function obtained by component $E^{d}(1)$ of complex discrete spectrum:

$\varphi_{o}=\left\{\begin{array}{l}\arctan \frac{\operatorname{Im}\left(E^{d}(1)\right)}{\operatorname{Re}\left(E^{d}(1)\right)} \quad ; \operatorname{Re}\left(E^{d}(1)\right) \geq 0 \\ \pi+\arctan \frac{\operatorname{Im}\left(E^{d}(1)\right)}{\operatorname{Re}\left(E^{d}(1)\right)} \quad ; \operatorname{Re}\left(E^{d}(1)\right)<0\end{array}\right.$

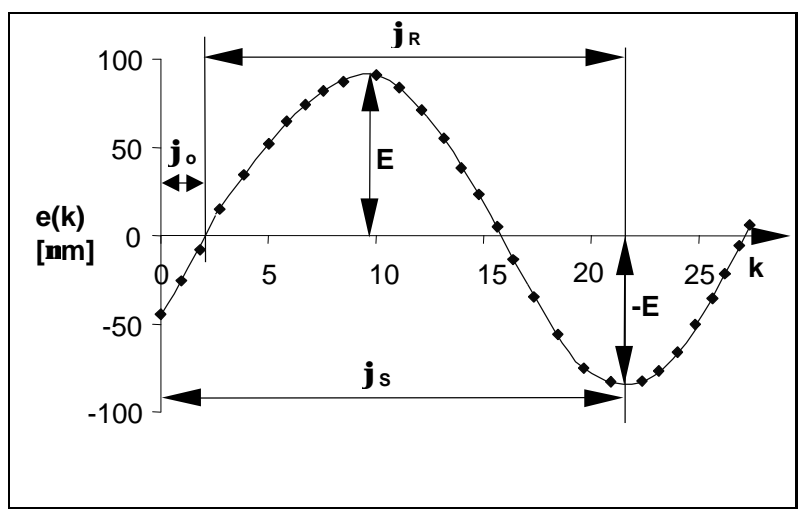

Figure 14: Determination of rotating angle $\varphi_{R}$

\section{References}

[1] A. Ravishankar Rao, "Future directions in industrial machine vision: a case study of semiconductor manufacturing applications ", Image and Vision Computing 14, 1993, 3-19

[2] O. Mohtadi and J. Sanz, "Recent progress in industrial machine vision", Int. J. Robotics and automation, Vol 8 No 2, 1993, 44-66

[3] R. Chin, "Survey of automated visual inspection:1981 to 1987“, Comput. Vision, Graph. Image Process., Vol 41, 1988, 346-381

[4] A. Wallace, "Industrial applications of computer vision since 1982“, IEEE Proc.., Vol 135 No 3, 1988, 117-136

[5] T.S. Newman, A.K. Jain, "A survey of automated visual inspection", Computer Vision and Image Understanding 61, 1995, 231-262

[6] M. Sonka, V. Hlavac, R. Boyle, Image processing , Analysis and Machine Vison, PWS Publishing, 1999

[7] E.R. Davies, Machine vision: theory, algorithms, practicalities, Academic Press, London 1990

[8] W.H. Press, S.A. Teulkolsky, W.T. Vetterling, B.P. Flannery, Numerical recipies in $C$, Cambridge university press, Cambridge, 1996

[9] P.L. Rosin, "A note on the least squares fitting of ellipses", Pattern Recognition Letters 14, 1993, 799-808

[10] Muhammad J. Mirza, Kim L. Boyer, "Performance evaluation of a class of M-estimators for surface parameter estimation in noisy range data", IEEE Transactions on Robotics and Automation 1, 1993, 75-85

[11] P. Meer, D. Mintz, A. Rosenfeld, D.Y. Kim, "Robust regression methods for computer vision", International Journal of Computer Vision 1, 1991, 59-70 